\title{
Multiple Forms of Facilitation Produced by Aversive Tentacular Stimuli in Cerebral Ganglion Sensory Neurons of Aplysia
}

\author{
Kent K. Fitzgerald ${ }^{1,2}$ and Thomas J. Carew ${ }^{1,3,4}$ \\ Departments of ${ }^{1}$ Psychology and ${ }^{3}$ Biology \\ Yale University \\ New Haven, Connecticut 06520
}

\begin{abstract}
Aversive tentacular stimuli can produce both nonassociative and associative modification of head-waving behavior of Aplysia. Sensory neurons (the $J / K$ cluster SNs) in the cerebral ganglion of Aplysia constitute an afferent pathway for aversive stimuli of the anterior tentacles. We used intracellular recording to examine plasticity in these neurons, particularly side-specific or site-specific alterations, which may be involved in mediating aspects of the learning induced by tentacle shock. The results of these experiments indicate that the $J / K$ SNs exhibit several forms of plasticity: (1) Post-tetanic potentiation (PTP); (2) heterosynaptic facilitation; (3) activity-dependent synaptic facilitation; and (4) side-specific spike broadening. Furthermore, by activating SNs directly to produce PTP and producing heterosynaptic facilitation with tentacular stimuli that were either inside or outside the receptive field of individual SNs, it was possible to dissect and analyze the differential contribution of intrinsic SN activity and heterosynaptic modulation to activity-dependent facilitation induced by behaviorally relevant stimuli. Collectively, these data raise the possibility that plasticity in primary afferent SNs may be involved in US processing during learning induced by tentacle stimulation.
\end{abstract}

${ }^{2}$ Present address: Center for Neural Science, New York University, New York, New York 10003.

${ }^{4}$ Corresponding author.

\section{Introduction}

The behavioral experiments described by Fitzgerald et al. (1997) demonstrate that an aversive stimulus, electric shock to the anterior tentacles, is effective in producing both nonassociative and associative learning in the head-waving behavior of Aplysia californica, a marine mollusk whose relatively simple nervous system is amenable to cellular analysis. To begin to explore the cellular mechanisms that may contribute to this form of learning, we conducted experiments to examine plasticity in the pathway that carries unconditioned stimulus (US) information. Specifically, we studied the effects of anterior tentacle shock in mechanoafferent sensory neurons (SNs) in the $J$ and $K$ clusters of the cerebral ganglion. These SNs, first characterized by Rosen and colleagues (1979), have somata that are located in two well-defined clusters in each cerebral hemiganglion: the lateral J cluster and the medial $\mathrm{K}$ cluster, totaling $\sim 200$ cells. The $\mathrm{J} / \mathrm{K}$ cells extend axonal processes in the cerebral ganglion nerves, which innervate the lips and tentacles. J/K neurons are normally silent and do not appear to receive any synaptic input. Individual neurons respond to tactile or electrical stimuli within restricted receptive fields on the tentacles with brief, accommodating bursts of action potentials. The $\mathrm{J} / \mathrm{K}$ cells form monosynaptic connections on tentacle motor neurons (MNs) located in the B cluster of the cerebral ganglion (Fredman and Jahan-Parwar 1977).

In many respects, the $\mathrm{J} / \mathrm{K}$ cells are homologous to other identified SN populations in Aplysia (see Clatworthy and Walters 1994), sharing similar response properties and anatomic organization. However, unlike other Aplysia SNs, the J/K cells exhibit heterogeneity in their anatomy, connectivity, and pharmacological modulation. A subset of the $\mathrm{J} / \mathrm{K}$ cells, designated the interganglionic cere-

LEARNING \& MEMORY 3:376-388 @ 1997 by Cold Spring Harbor Laboratory Press ISSN1072-0502/97 \$5.00

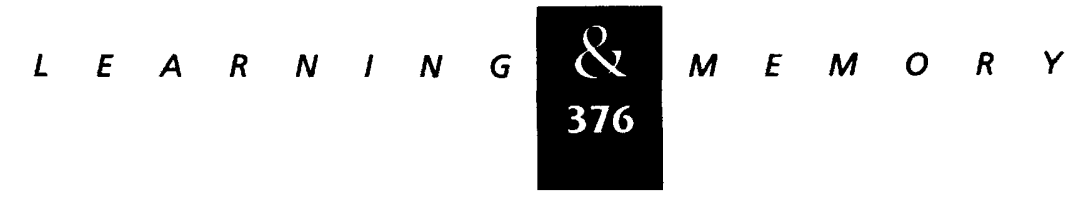


bral-buccal mechanoafferents (ICBMs), have a characteristic axonal process in the cerebral-buccal connective and innervate dual receptive fields in the perioral zone and the buccal mass (Rosen et al. 1982). On the basis of on their synaptic connectivity, the ICBMs are believed to be involved in appetitive functions, whereas other J/K SNs probably serve a defensive function. Serotonin also produces heterogeneous effects in the $\mathrm{J} / \mathrm{K}$ cells; in individual neurons, serotonin produces either spike broadening, accompanied by synaptic facilitation, or spike narrowing, with concomitant synaptic inhibition (Rosen et al. 1989).

Several lines of evidence suggest that the $\mathrm{J} / \mathrm{K}$ SNs are an important input pathway mediating the effects of aversive anterior tentacle USs, and thus their modulation may have important consequences for learning produced by such stimuli. First, the J/K SNs innervate the anterior tentacles with overlapping receptive fields, and it is highly likely that shock stimuli delivered anywhere on the tentacles will activate at least one, and probably several, of these cells. Thus, the J/K neurons, along with SNs in other populations (such as the pleural ganglion SNs), constitute a major input pathway for tactile stimuli of the anterior tentacles. Second, as has been observed by Walters (1987b), Aplysia central mechanoafferent neurons exhibit properties that suggest they may serve a nociceptive function. In particular, these SNs respond in a graded manner to aversive stimuli within a wide dynamic range and are maximally responsive to very strong or injurious stimuli. Thus, the response characteristics of these neurons appear appropriate to mediate the effects of aversive stimuli. As described above, a subset of the J/K cells (the ICBM neurons) have response properties that indicate an appetitive, rather than aversive function (Rosen et al. 1982). However, this subset comprises only a small fraction ( $10-12$ cells) of the entire $\mathrm{J} / \mathrm{K}$ population. Finally, recent evidence by Raymond and Byrne (1994) shows that J cluster cerebral SNs exert synaptic modulatory influences on sensory and interneuronal elements of the tail withdrawal circuit. The kinds of modulation produced by the $J$ cells, such as an increase in excitability in pleural SNs, are similar to the effects produced by sensitizing stimuli. These findings support the role of cerebral SNs as a pathway for aversive stimuli. Thus, it seems likely that the $\mathrm{J} / \mathrm{K}$ cells constitute an input pathway for aversive tentacle stimuli and, therefore, that plasticity in these neurons may contribute importantly to learning.
An additional motivation for studying these cells was to address the more general issue of learning-related plasticity in different populations of Aplysia SNs. Central mechanoafferent SNs of Aplysia have proven to be valuable model systems for investigating the neural mechanisms of learning and memory. Specifically, heterosynaptic facilitation in SNs is thought to underlie behavioral sensitization, whereas additional, activity-dependent enhancement of facilitation contributes to classical conditioning (for review, see Castellucci and Schacher 1990; Byrne et al. 1991; Marcus et al. 1994; Byrne and Kandel 1996). Although these forms of learning-related plasticity have been analyzed extensively in SNs of the abdominal and pleural ganglia (which participate in defensive withdrawal reflexes such as gill/siphon withdrawal and tail withdrawal, respectively), less research has examined the possible role of other $\mathrm{SN}$ populations in learning. Thus, it was of interest to determine whether the forms of plasticity described above are a general feature of Aplysia SNs and, in addition, whether similar cellular mechanisms underlie these forms of plasticity in different types of SNs.

In this paper, several forms of modulation were examined in the cerebral ganglion SNs, including synaptic facilitation and spike broadening, produced by both heterosynaptic and activity-dependent processes. Because the behavioral results described by Fitzgerald et al. (this issue) indicate that learning produced by anterior tentacle stimulation is dependent on the location of the US, it was of particular interest in these cellular experiments to examine types of plasticity that exhibit. spatial specificity. We identified two types of plasticity that are influenced by the location of an aversive US: side-specific modulation of spike duration and site-specific activity-dependent synaptic facilitation. These diverse forms of plasticity provide attractive candidate mechanisms that may contribute to US processing during learning induced by tentacular stimulation.

\section{Materials and Methods}

\section{ANIMALS}

Wild-collected adult $A$. californica (150-300 grams) were obtained from commercial suppliers on the Pacific coast (Marinus, Pacific Biomarine, or Alacrity). In the laboratory, animals were maintained in aquaria (1000 liters) filled with aerated

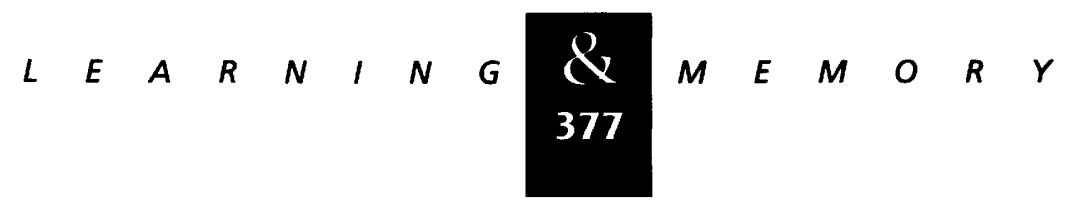


artificial sea water (Instant Ocean, Aquarium Systems, Mentor $\mathrm{OH}$ ) at $15^{\circ} \mathrm{C}$. Animals were fed seaweed (Nagai Sushinori, Japan, $\sim 0.75$ gram daily).

\section{INTRACELLULAR RECORDING}

Intracellular recordings were performed in a reduced preparation consisting of the anterior tentacles, attached via the nerves C1 (upper labial) and C2 (anterior tentacular) to the cerebral, pleural, and pedal ganglia (Fig. 1A). Aplysia were anesthetized for dissection by injection of $\sim 150 \mathrm{ml}$ of $\mathrm{MgCl}_{2}$ (364 mM, with $10 \mathrm{~mm}$ Tris buffer at $\mathrm{pH}$ 7.6). During dissection, the preparation was bathed in physiological saline consisting of $460 \mathrm{~mm} \mathrm{NaCl}, 55$ $\mathrm{mm} \mathrm{MgCl}_{2} ; 11 \mathrm{~mm} \mathrm{CaCl}_{2}, 10 \mathrm{~mm} \mathrm{KCl}$, and $10 \mathrm{~mm}$ Tris buffer ( $\mathrm{pH}$ 7.6). The preparation was placed in a 55-mm-diam. plastic dish, secured with pins to the floor of the dish, and perfused continuously with saline during recording. Experiments were conducted at room temperature $\left(20^{\circ} \mathrm{C}\right)$. Glass microelectrodes (10-20 M $\Omega$, filled with $3 \mathrm{~m}$ potas-
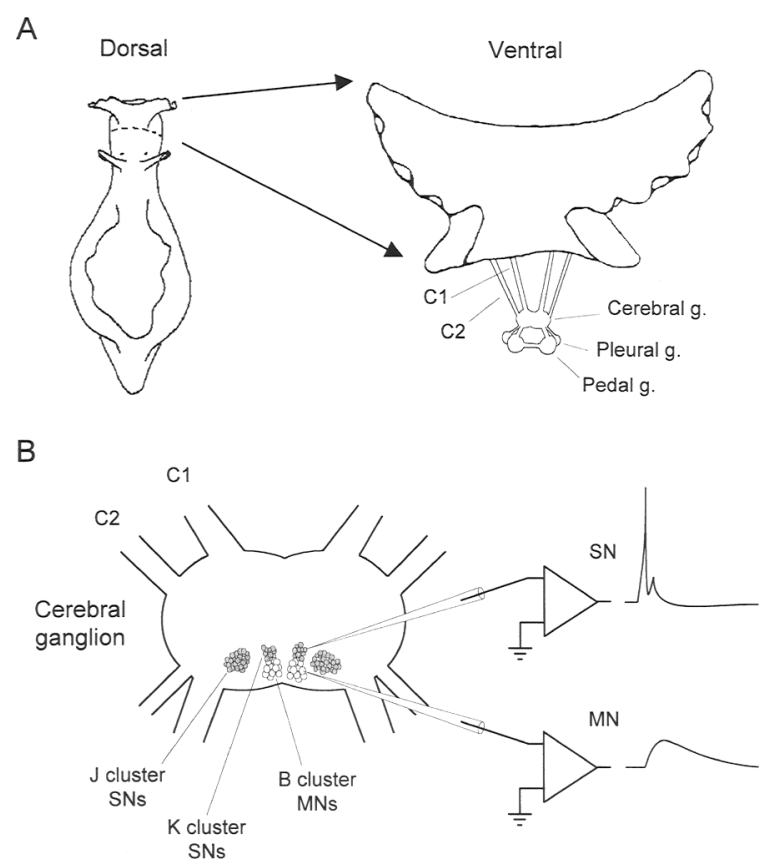

Figure 1: Reduced preparation used for cellular experiments. (A) To investigate sensory input from the anterior tentacles, a reduced preparation was used that consisted of the tentacles, attached via nerves $\mathrm{C} 1$ and $\mathrm{C} 2$ to the cerebral ganglion. The pleural and pedal ganglia were included in some experiments. $(B)$ Relative locations of the $J$ and $K$ cluster SNs (shaded) and their postsynaptic targets, the B cluster MNs. sium acetate or $3 \mathrm{~m} \mathrm{KCl}$ ) were used for intracellular recordings.

For measurements of action potential duration, 1.0 to 1.5 -msec pulses of depolarizing current were used to elicit spikes. Brief pulses were used so that the peak of the action potential occurred after the offset of the current pulse and, thus, measurement of spike duration was not contaminated by the external depolarization. Action potential duration was measured as the time from the peak of the action potential until membrane potential fell to $20 \%$ of peak amplitude.

Tentacle shock was delivered with bipolar electrodes identical to those used in the behavioral experiments (Fitzgerald et al. 1997). The electrode probe was positioned by a mechanical micromanipulator to maintain the electrodes in firm contact with the skin of the anterior tentacles. Because the tentacles were secured by pins, the position of the stimulus probe on the tentacles remained constant.

\section{SYNAPTIC EXPERIMENTS}

Experiments examining changes in the synaptic output of the cerebral ganglion SNs were conducted by pairwise recording of the monosynaptic connection from the SNs to the MNs of the cerebral ganglion B cluster (Fig. 1B; Rosen, et al. 1979). The resting potential of MNs was typically -45 to $-50 \mathrm{mV}$. To allow measurement of excitatory postsynaptic potential (EPSP) amplitude uncontaminated by action potentials, MNs were hyperpolarized to $-80 \mathrm{mV}$ at the beginning of each experiment and held with a constant level of injected current. Single SN spikes, which produced monosynaptic EPSPs in the MNs, were elicited by 10- to 20-msec pulses of depolarizing current.

Once a monosynaptic connection was identified, two or more treatments were examined in sequential phases (in random order). Each phase of an experiment lasted $15 \mathrm{~min}$ and consisted of a total of 15 tests: 5 pre-tests and 5 post-tests were conducted at $30-\mathrm{sec}$ intervals, and 5 subsequent post-tests were performed at 2-min intervals. A period of at least $30 \mathrm{~min}$ of recovery was allowed to elapse between successive phases in an experiment.

\section{STATISTICAL ANALYSIS}

Group data are expressed as means \pm standard errors of the mean. For analysis, EPSP amplitudes at

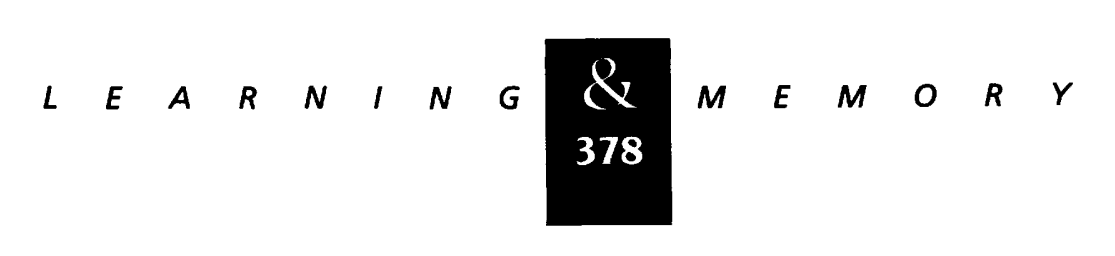


each post-test were converted to difference scores (amplitude at each post-test minus amplitude at the last pre-test). A two-way ANOVA, with time as a within-subject factor, was used to examine statistical differences between groups. Fisher's protected least significant difference test (PLSD) was used for specific between-group comparisons. To determine whether post-test EPSP amplitudes were facilitated relative to baseline, a $t$-test for related means was used to test whether post-test difference scores differed significantly from zero. Correlations were evaluated by Pearson's test. The standard level of significance was $P=0.05$. All probability values are two-tailed.

\section{Results}

\section{ACTIVITY-DEPENDENT SYNAPTIC FACILITATION IN CEREBRAL GANGLION SNS}

The objective of this experiment was to determine whether the J/K neurons exhibit activity-de- pendent synaptic facilitation. This form of plasticity produces enhanced facilitation of synaptic output that is specific to activated SNs (Hawkins et al. 1983; Walters and Byrne 1983) and is thus a possible mechanism for encoding the specific location of stimuli that activate SNs. Facilitation was examined at the monosynaptic connection between $\mathrm{J} / \mathrm{K}$ neurons and B cluster MNs. Activity-dependent synaptic facilitation in other Aplysia SNs has been demonstrated previously using a cellular training procedure analogous to classical conditioning: A train of $\mathrm{SN}$ action potentials, representing the conditioned stimulus (CS) was paired with a heterosynaptic-facilitating US, such as tail or nerve shock (Hawkins et al. 1983, Walters and Byrne 1983). Using a similar procedure, we compared three treatments (Fig. 2) (1) Shock alone consisted of a single 200 -msec shock at $65 \mathrm{~V}$ and $60 \mathrm{~Hz} \mathrm{AC}$ to the ipsilateral anterior tentacle. In this example, shock was applied outside of the receptive field of the $\mathrm{SN}$ being recorded. (2) Activity alone consisted of a train of 20-msec pulses of intracellular depolarizing current, delivered at $10 \mathrm{~Hz}$ for $1 \mathrm{sec}$. A train of pulses was used because Aplysia SNs exhibit ac-

\section{A Shock alone}

$\mathrm{Vm}$

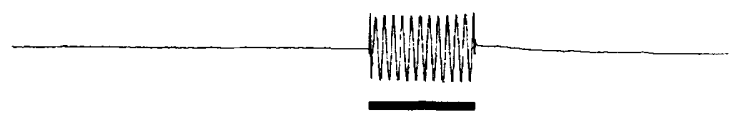

\section{B Activity alone}

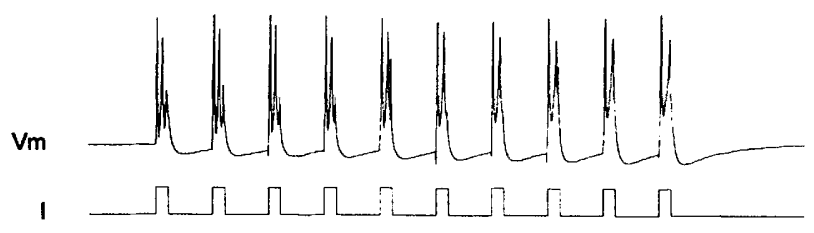

C Pairing: shock + activity

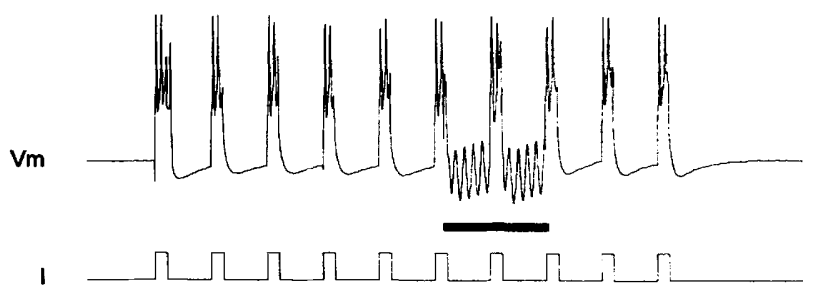

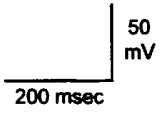
polarizing current pulses. The onset of shock occurred $550 \mathrm{msec}$ after the beginning of the pulse train.

Figure 2: Training procedures used to examine activity-dependent synaptic facilitation. The intracellularly recorded response of a $\mathrm{SN}(\mathrm{Vm})$ to each of the three treatments is shown. $(A)$ Tentacle shock ( $200 \mathrm{msec}$ duration) is indicated by the horizontal bar. The sinusoid is the shock artifact. Because shock was applied outside of the receptive field, the SN was not activated. (B) The lower trace (I) indicates intracellular current pulses. Activity alone consisted of a 1-sec, 10$\mathrm{Hz}$ train of 20-msec duration depolarizing pulses. In this experiment, each pulse elicited two $\mathrm{SN}$ action potentials (top trace). (C) Pairing consisted of shock applied during a train of de-

$$
\begin{array}{lllllllllllllll}
L & E & A & R & N & I & N & G & \begin{array}{l}
\mathcal{Z} \\
379
\end{array} & M & E & M & O & R & Y
\end{array}
$$


commodation of firing to long depolarizations; a train of brief pulses, however, reliably elicited at least one action potential per pulse. (3) Pairing of shock and activity was arranged so that the shock occurred in the middle of the pulse train. The order of the three treatments was randomized. Whenever possible (in 10 of 12 experiments), all three conditions were examined in a single preparation.

The synaptic facilitation produced by these training procedures is shown in Figure 3. For statistical analysis, synaptic facilitation was measured as the difference in EPSP amplitude between each post-test and the last pretest prior to training. Shock alone $(n=11)$ produced no significant change in EPSP amplitude. Activity alone $(n=12)$ produced significant synaptic facilitation at 0.5 , 2.5 , and $4.5 \mathrm{~min}$ after training $[0.5 \mathrm{~min}, t(11)=$ $3.95, P<0.005 ; 2.5 \mathrm{~min}, t(11)=2.94, P<0.02 ; 4.5$ $\min , t(11)=3.34, P<0.01]$. This facilitation is likely attributable to post-tetanic potentiation, a fairly ubiquitous form of homosynaptic facilitation that has been observed previously in the $\mathrm{J} / \mathrm{K}$ cells (Walters and Byrne 1984). Pairing of shock and activity $(n=10)$ induced activity-dependent synaptic facilitation at $0.5,2.5$, and $4.5 \mathrm{~min}$ after training $[0.5 \mathrm{~min}, t(9)=3.60, P<0.01 ; 2.5 \mathrm{~min}, t(9)=2.59$, $P<0.05 ; 4.5 \mathrm{~min}, t(9)=3.57, P<0.01]$. This facilitation was substantially greater in amplitude and duration than facilitation produced by the other treatments. An ANOVA revealed a significant effect of treatment $[F(2,30)=3.56, P<0.05]$. Post hoc comparisons showed that pairing produced greater facilitation than either activity alone (Fisher's PLSD, $P=0.05)$ or shock alone $(P<0.02)$. Furthermore, there was a highly significant interaction of treatment and time after training $[F(8,30)=8.59$, $P<0.0001$ ], indicating that the time course of facilitation differed between treatments. It appears that post-tetanic potentiation was greatest immediately after training, whereas activity-dependent synaptic facilitation built up gradually, reaching a maximum level $4.5 \mathrm{~min}$ after training. These results show that the cerebral ganglion SNs exhibit an intrinsic form of synaptic facilitation (PTP) that is significantly enhanced by pairing with tentacle shock.

\section{ACTTVITY-DEPENDENT SYNAPTIC FACILITATION INDUCED BY A SINGLE AVERSIVE STIMULUS}

The process of activity-dependent synaptic facilitation in Aplysia SNs was proposed initially as a mechanism for encoding the conjunction of two separate stimuli, the CS and US, during classic conditioning (Hawkins et al. 1983; Walters and Byrne 1983). The experiment described above (Fig. 3)
A

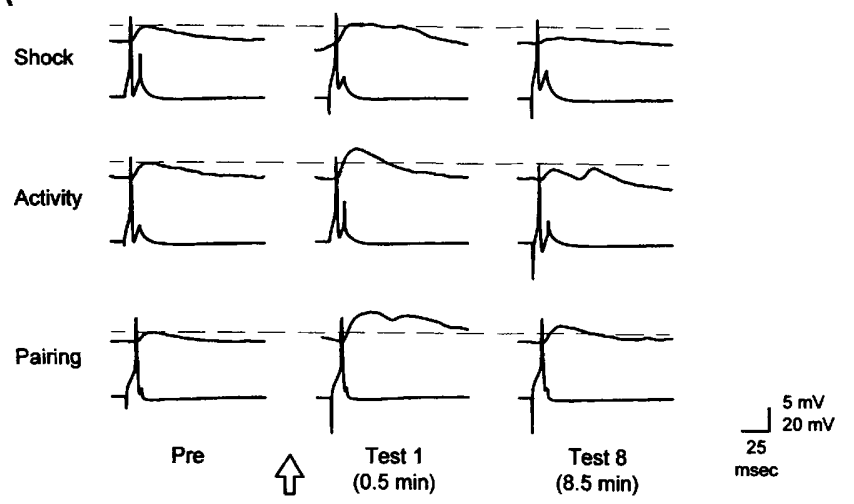

B

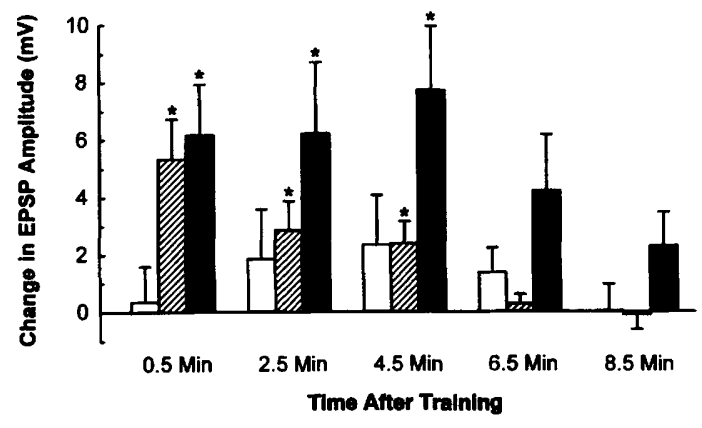

Figure 3: Activity-dependent synaptic facilitation in cerebral ganglion SNs. $(A)$ Example of synaptic modulation produced by three treatments. All traces are from the same pair of cells. EPSPs in B cluster MNs (top trace in each pair) were produced by single action potentials elicited in SNs (bottom trace). (Pre) The last of five pretests. The broken line indicates the amplitude of the pretest EPSP. Shock alone produced a slight elevation of the EPSP at Test 1 . The EPSP returned rapidly to baseline amplitude and then continued to diminish, presumably because of homosynaptic depression. Activity alone produced more substantial facilitation, which is attributable to PTP. However, facilitation was no longer evident by 8.5 min. Pairing produced facilitation that lasted at least until Test 8, $8.5 \mathrm{~min}$ after training. $(B)$ In this summary, facilitation is expressed as a change in EPSP amplitude (Test minus Pre). Asterisks indicate significant facilitation above baseline $(P<0.05)$. Pairing (shaded bars) produced significantly greater facilitation than shock (open bars) or activity (hatched bars) (see text).

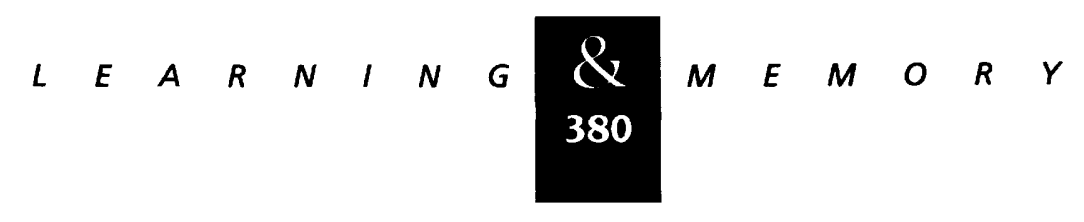


demonstrated activity-dependent synaptic facilitation using a procedure that has been regarded as an analog of classic conditioning: the explicit pairing of $\mathrm{SN}$ activation with an aversive US. However, in the behavioral experiments presented in the preceding paper (Fitzgerald et al. 1997), learning was produced by an aversive stimulus, anterior tentacle shock, presented alone. To ascertain whether activity-dependent synaptic facilitation might contribute to this form of learning, we wished to investigate whether the US used behaviorally (tentacle shock) can by itself induce activity-dependent synaptic facilitation in the SNs. Previous work examining Aplysia pleural ganglion SNs has shown that a single, unpaired stimulus that activates a SN can evoke activity-dependent synaptic facilitation by simultaneously producing SN activity and inducing heterosynaptic facilitation. Furthermore, it has been proposed that facilitation induced in this manner provides a mechanism for site-specific sensitization (Walters 1987a,b). In the next experiment we examined whether a single tentacleshock US can induce activity-dependent facilitation in the cerebral ganglion SNs.

To determine whether the J/K SNs undergo activity-dependent synaptic facilitation in response to cutaneous activation by tentacle shock, the effects of shock either within or outside the SN receptive field were compared. While recording a monosynaptic connection, shock was delivered either to the site on the ipsilateral anterior tentacle that produced maximal SN activation $(n=18)$, or a control site $(n=16)$ on the same tentacle that did not activate the $\mathrm{SN}$. Stimulation within the receptive field produced a burst of $\sim 10-15$ action potentials. Both conditions were compared sequentially (in random order) in the same experiment when possible (in 10 of 24 preparations).

The results of the first experiment are shown in Figure 4. Shock within the receptive field produced significant facilitation of EPSP amplitude at $0.5,2.5,4.5$, and $6.5 \mathrm{~min}$ after shock $[0.5 \mathrm{~min}$, $t(17)=3.78, P<0.01 ; 2.5 \mathrm{~min}, t(17)=3.17, P<$ $0.01 ; 4.5 \mathrm{~min}, t(17)=2.91, P<0.01,6.5 \mathrm{~min}$, $t(17)=2.85, P<0.05]$. Shock alone, outside of the receptive field, produced only very modest heterosynaptic facilitation $[0.5 \mathrm{~min}$ after shock, $t(15)=$ 2.54, $P<0.05$ ]. An ANOVA indicated that shock within the receptive field produced significantly greater synaptic facilitation than shock outside [effect of location of shock, $F(1,32)=4.32, P<0.05$ ]. Thus, a brief shock within the receptive field of a $\mathrm{SN}$ is capable of inducing activity-dependent synaptic facilitation. The time course of this facilitation (Fig. 4B) is quite similar to that produced by the pairing of shock and intracellular depolarization in the previous experiment (cf. Fig. 3B). However, the overall level of facilitation produced by receptive field shock was $\sim 40 \%$ less than that produced by pairing, a difference that may be attributable to the smaller number of action potentials elicited by shock, as compared to intracellular depolarization.

To further test the hypothesis that receptive-

B

A
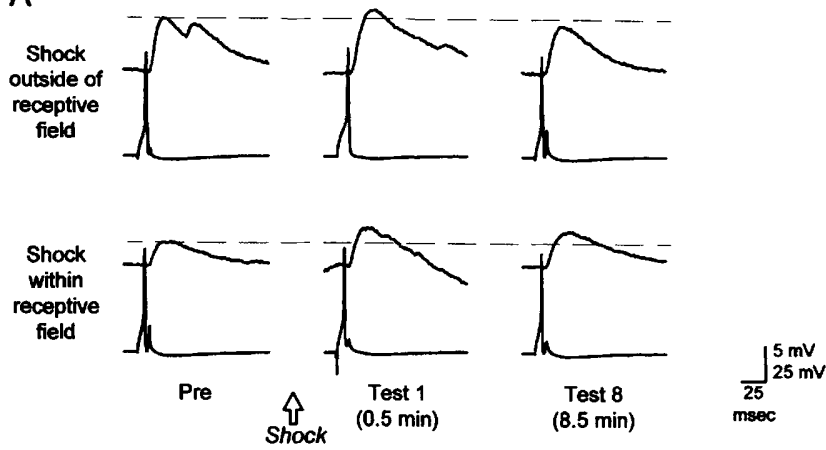

Figure 4: Receptive-field activation induces activity-dependent synaptic facilitation in SNs. (A) EPSPs in B cluster MNs (top trace in each pair) were produced by single action potentials elicited in SNs (bottom trace). Shock outside of the receptive field produces transient facilitation, whereas shock within the receptive field produces greater and longer-lasting enhancement. (B) Facilitation is expressed as a change in EPSP amplitude (Test minus Pre). Asterisks $\left(^{*}\right)$ indicate significant facilitation above baseline $(P<0.05)$. (Open bars) Shock outside receptive field; (shaded bars) shock within receptive field. Note that the temporal profile of facilitation produced by receptive-field activation resembles that produced by experimental pairing of shock and activity (cf. Fig. 3).

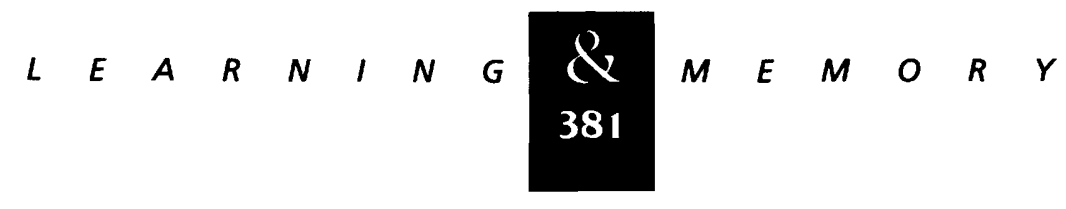


field activation produces activity-dependent synaptic facilitation, two additional analyses were conducted. Activity-dependent synaptic facilitation is produced by the interaction of $\mathrm{Ca}^{2+}$ influx (caused by action potentials) with the biochemical cascade of heterosynaptic facilitation (Abrams 1985; Ocorr et al. 1985; Yovell and Abrams 1992). Thus, the degree of facilitation would be expected to be a graded function of the number of presynaptic action potentials. To test this prediction, a post hoc analysis of the data from the receptive-field experiment was conducted, to determine whether cells that were more strongly activated by shock exhibited a greater level of synaptic facilitation (although the shock stimulus was constant, individual SNs varied in their responsiveness). Because action potentials occurring during the stimulus were often obscured by the shock artifact, action potentials were counted in a 275-msec interval following stimulus offset. There was a strong positive correlation between the number of presynaptic action potentials and the degree of synaptic facilitation $[R=0.671, F(1,16)=13.1, P=0.002]$. Thus, the number of action potentials elicited by receptivefield shock accounts for $45 \%$ of the variability in the degree of facilitation produced by shock. Other factors contributing to facilitation may include variation between preparations in the efficacy of shock and the degree of activation of heterosynaptic facilitatory elements.

\section{A1 SN allowed to fire}

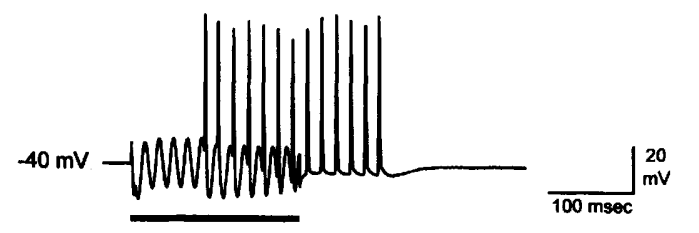

\section{A2 $\mathrm{SN}$ hyperpolarized}

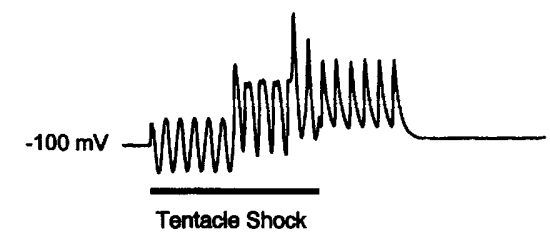

If shock within the receptive field of a $\mathrm{SN}$ does induce activity-dependent synaptic facilitation, then it should be possible to diminish facilitation by blocking SN spike activity. To test this prediction, the facilitation produced by tentacle shock within the receptive field of the $\mathrm{SN}$ was examined under two conditions: The SN was either allowed to fire in response to tentacle shock (as in the previous experiment), or it was deeply hyperpolarized with injected current (to below $-80 \mathrm{mV}$ ) to prevent or diminish firing during shock. Although it was not possible to block the generation of action potentials completely, the amplitude and number of action potentials recorded in the soma was substantially reduced by hyperpolarization (Fig. 5A). These two conditions were compared using a within-group design $(n=5)$, with the order of treatments randomized. When allowed to fire, $\mathrm{SNs}$ exhibited significant synaptic facilitation at 0.5 and $2.5 \mathrm{~min}$ after shock [Fig. $5 \mathrm{~B} ; 0.5 \mathrm{~min}, t(4)=3.97$, $P<0.02 ; 2.5 \mathrm{~min}, t(4)=3.22, P<0.05$ ]. When hyperpolarized, the same cells did not exhibit significant facilitation. Thus, the facilitation produced by receptive-field shock is dependent on SN firing.

In summary, these experiments show that (1) greater synaptic facilitation is produced by tentacle shock within the receptive field of an SN, thereby activating the cell, than shock outside the receptive field of the cell; (2) when shock is delivered within the receptive field, thereby activating the

B

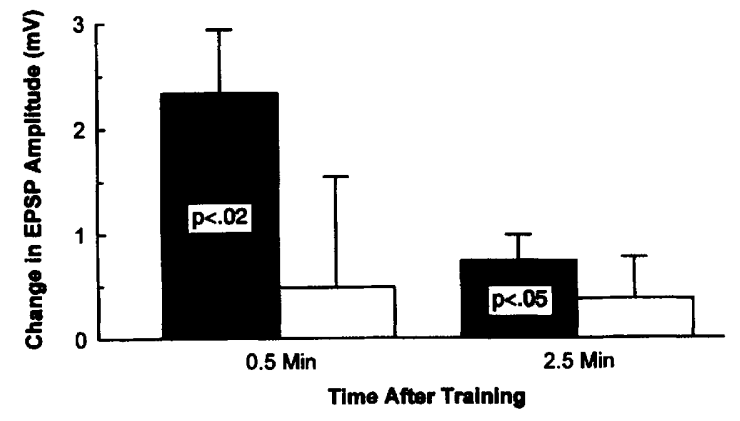

Figure 5: Activity-dependent synaptic facilitation is blocked by SN hyperpolarization. (A) Examples of SN responses to training stimuli. In both cases, shock was delivered within the receptive field. Normally, tentacle shock produced a burst of action potentials ( 1 ). When the SN was hyperpolarized with injected current (2), action potentials were blocked before they reached the soma. $(B)$ Synaptic facilitation produced by tentacle shock within the receptive field is expressed as a change in EPSP amplitude (Test minus Pre). Probability values indicate significant facilitation above baseline. Hyperpolarization of the SN (open bars) prevents the synaptic facilitation that is normally produced by shock (shaded bars).

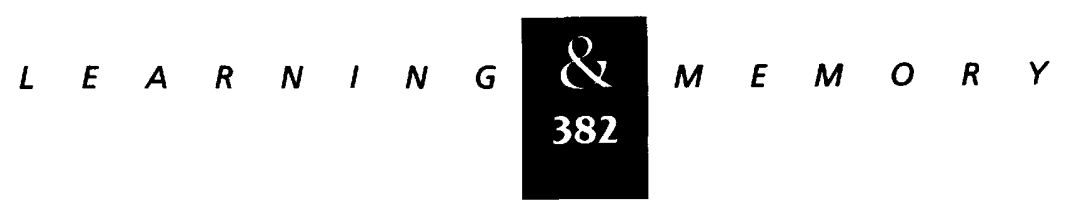


$\mathrm{SN}$, the degree of facilitation is correlated with the number of action potentials elicited by shock; and (3) receptive-field activation does not produce facilitation when the SN soma is hyperpolarized. This latter observation also rules out the possibility that stimulation within the receptive field of a SN produces greater heterosynaptic facilitation per se (independent of activation of the $\mathrm{SN}$ ), as an identical site of tentacle stimulation was employed under both normal and hyperpolarized conditions.

\section{MODULATION OF ACTION POTENTIAL DURATION}

Several mechanisms have been shown to contribute to synaptic facilitation in Aplysia SNs, one of which is spike broadening, which results from a decrease in $\mathrm{K}^{+}$currents and allows a greater influx of $\mathrm{Ca}^{2+}$ during the action potential, thus increasing neurotransmitter release (Klein and Kandel 1978; for review, see Byrne and Kandel 1996). To determine whether the synaptic facilitation induced by anterior tentacle shock involves spike broadening, action potential duration was measured in $\mathrm{J} / \mathrm{K}$ cluster SNs before and after tentacle shock. Additionally, because the behavioral effects of training with anterior tentacle shock are side specific, the effects of ipsilateral and contralateral shock were compared. Because these experiments were intended to examine side-specific differences produced by heterosynaptic modulation, shock was applied to sites on the tentacles that did not activate the SN from which the recording was obtained.

In a pilot study, it was determined that spike broadening in response to tentacle shock was not observed readily unless SNs first underwent frequency broadening. Frequency broadening (Fig. 6A1) refers to a progressive increase in action potential duration that occurs when spikes are elicited repeatedly at a short interval (e.g., $1 \mathrm{~Hz}$ ) and presumably results from the cumulative inactivation of one or more voltage-dependent $\mathrm{K}^{+}$currents (Ma and Koester 1996). Therefore, in this experiment, the effects of shock were examined by first activating the cell at $1 \mathrm{~Hz}$ for $30-120 \mathrm{sec}$, until frequency broadening reached a plateau level, and then applying shock. This manipulation was used as an analytical tool to permit measurement of subtle changes in action potential duration and was not intended to realistically mimic physiological activation. It is not known whether these SNs undergo frequency broadening in response to cutaneous activation in vivo. Under these conditions, we found that tentacle shock, applied ipsilaterally, produced considerable additional spike broadening (superimposed on frequency broadening; Fig. 6A2). To a lesser extent, contralateral shock also produced additional broadening (Fig. 6A3). Because the extent of frequency broadening varied among cells, for statistical analysis action potential
A

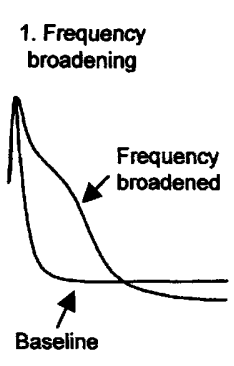

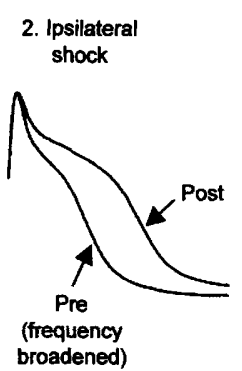

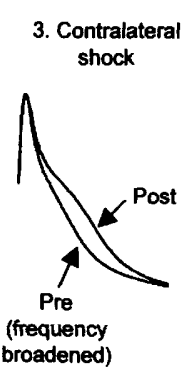

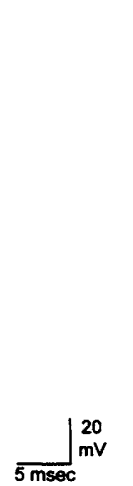

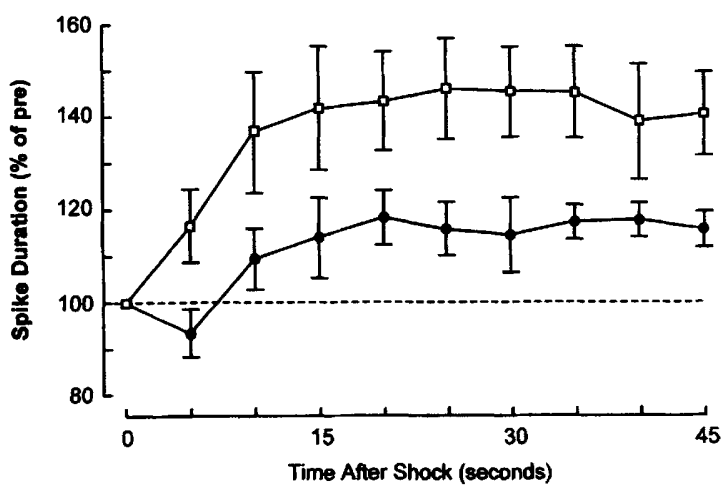

Figure 6: Tentacle shock produces side-specific spike broadening in cerebral ganglion SNs. (A) Examples of spike broadening produced by repeated activation and tentacle shock. In each pair of traces, two action potentials, recorded before and after the indicated treatment, are superimposed. SNs first were activated repeatedly by intracellular depolarization at $1 \mathrm{~Hz}$, which produced frequency broadening (1). Subsequently, shock to either the ipsilateral (2) or contralateral (3) anterior tentacle produced additional spike broadening. (B) Summary of five experiments. Spike duration, which was measured at 5-sec intervals (i.e., for every fifth action potential), is expressed as a percentage of the last spike prior to shock. Ipsilateral shock $(\square)$ produced significantly greater broadening than contralateral shock $(\bullet)$.

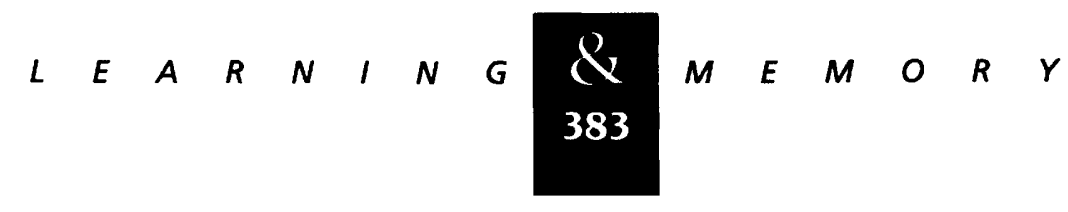


duration was expressed as a percentage of the duration of the frequency-broadened spike immediately prior to shock. A two-way ANOVA indicated that ipsilateral shock produced significantly greater broadening than contralateral shock (Fig. 6B; effect of side of shock, $F(1,8)=5.64, P<0.05)$. There was no significant interaction between side of shock and time. We also observed that shock often produced transient spike narrowing, lasting for a few seconds, which is evident at the 5-sec time point in the group data for contralateral shock (Fig. 6B, solid circles).

The finding that spike broadening is observed only when superimposed on frequency broadening has two possible interpretations. First, it may be that the ionic current modulated by tentacle shock represents a fairly small proportion of the total membrane current during the falling phase of action potential. Thus, frequency broadening, which presumably results from the inactivation of one or more of the major voltage-dependent $\mathrm{K}^{+}$ currents, may serve to unmask the subtle modulation of a smaller current. Another possibility is that there is an actual permissive requirement for frequency broadening, possibly indicating the involvement of a $\mathrm{Ca}^{2+}$-dependent process, in spike broadening induced by tentacle stimulation, similar to that underlying activity-dependent synaptic facilitation. The "shoulder" of a broadened action potential is caused primarily by an inward $\mathrm{Ca}^{2+}$ current (Klein and Kandel 1978; Klein et al. 1982), and frequency broadening would therefore be expected to be accompanied by a large influx of $\mathrm{Ca}^{2+}$. It may be that the resulting increase in intracellular $\mathrm{Ca}^{2+}$ is a mechanistic prerequisite for the expression of tentacle shock-induced spike broadening. Therefore, the spike broadening that we have observed may reflect an activity-dependent process.

\section{Discussion}

\section{MULTIPLE FORMS OF PLASTICITY IN CEREBRAL GANGLION SNS}

In our experiments we have demonstrated several distinct forms of plasticity in the $\mathrm{J} / \mathrm{K}$ cluster SNs of the cerebral ganglion: homosynaptic facilitation [post-tetanic polentiation (PTP)], which is induced by individual SN activation; heterosynaptic facilitation, which is produced by tentacle shock; activity-dependent synaptic facilitation, which results from contiguous tentacle shock and SN activity; and side-specific spike broadening. A model circuit that could give rise to these three forms of plasticity is shown schematically in Figure 7. As indicated in Figure 7, we hypothesize that some of the effects of tentacle shock are mediated via facilitatory interneurons, as has been demonstrated for plasticity in other populations of Aplysia SNs (e.g., Hawkins 1981; Hawkins and Schacher 1989; Mackey et al. 1989). A number of facilitatory interneurons have been identified in

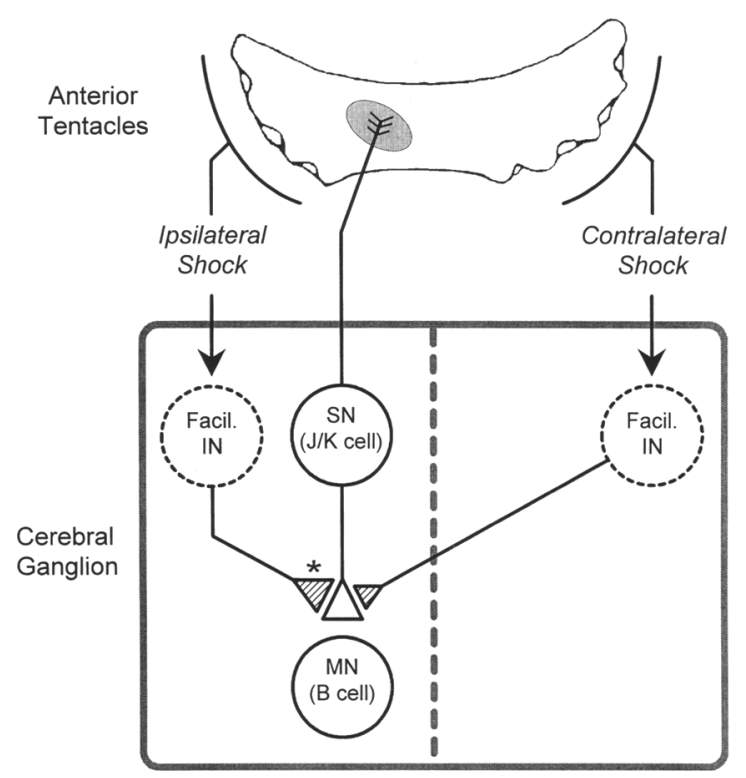

$$
\checkmark \text { Excitatory Synapse Facilitatory Synapse }
$$

Figure 7: Model circuit for forms of plasticity in cerebral ganglion SNs. Depicted is the neural circuitry proposed to underlie three forms of synaptic facilitation that we have observed in cerebral ganglion SNs. Circles with solid lines indicate identified neurons; circles with broken lines indicate cells that have not been identified but whose participation is implied by the present results. J/K SNs, which form monosynaptic connections to B cluster MNs, are activated by stimuli within their individual receptive fields on the tentacles (shaded ellipse). Individual activation of SNs produces homosynaptic facilitation (PTP). Tentacle shock produces widespread heterosynaptic modulation via the action of interneurons. This facilitation has a side-specific component: Ipsilateral shock produces greater facilitation than contralateral shock (indicated by different sized modulatory terminals). Finally, the contiguous activation of a SN simultaneously with tentacle shock induces activity-dependent synaptic facilitation (asterisk).

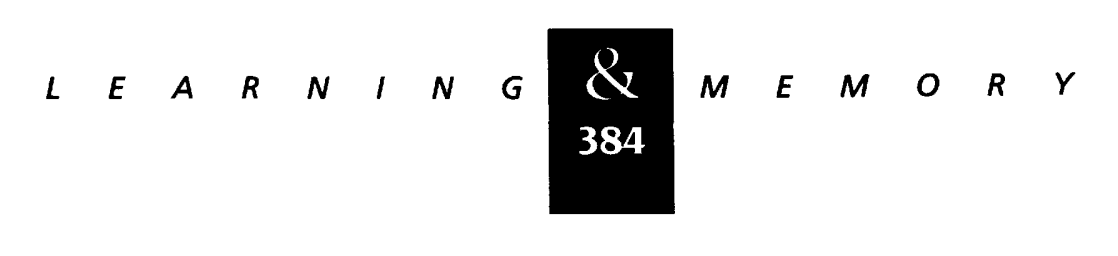


Aplysia, including one pair in the cerebral ganglion (Mackey et al. 1989), but it is not yet known whether any of these identified neurons produce facilitation in the J/K SNs. Another question that is open to exploration concerns the source of afferent input from the tentacles to facilitatory interneurons. The presence of such input is implied by the fact that tentacle shock induces heterosynaptic facilitation, but the actual input pathway is not known. It is possible that the $\mathrm{J} / \mathrm{K}$ cells themselves provide input to interneurons. In light of the evidence implicating the $\mathrm{J} / \mathrm{K}$ cells as a pathway for aversive tentacle stimuli, this seems quite likely. Thus, it is possible that the $\mathrm{J} / \mathrm{K}$ SNs participate in producing their own heterosynaptic modulation.

With the exception of PTP (Walters and Byrne 1984), the different types of plasticity that we have identified in our experiments have not been reported previously in cerebral ganglion SNs. Thus, the present results complement and extend an understanding of synaptic facilitation that has been obtained in other SN populations, and provide a basis for the comparative analysis of plasticity in different populations of Aplysia SNs. A novel finding in these experiments is that activity-dependent synaptic facilitation can be induced physiologically by a single, brief aversive stimulus to the anterior tentacles. Prior work examining the mechanisms of site-specific sensitization has shown that repeated training with shock (10 trials) within the receptive field of a $\mathrm{SN}$ induces activity-dependent facilitation (Walters 1987b); the present results demonstrate that in the cerebral ganglion SNs, a single, fairly mild stimulus is sufficient to produce this type of facilitation.

\section{ROLE OF SN ACTIVITY IN SYNAPTIC FACILITATION}

In our experiments, several manipulations have demonstrated that SN activity produces enhanced synaptic facilitation. First, $\mathrm{SN}$ activation by intracellular depolarization amplifies the facilitation that is produced by tentacle shock. Second, shock within the receptive field of a SN (causing the SN to fire) induces greater facilitation than shock elsewhere on the tentacles. Finally, the enhanced facilitation produced by receptive-field activation is blocked by intracellular hyperpolarization of the SN. These results can be interpreted as indicating that a process intrinsic to the SNs, presumably mediated by the influx of $\mathrm{Ca}^{2+}$ induced by action potentials, produces enhanced presynaptic facilitation. This is the mechanism of activity-dependent synaptic facilitation that has been demonstrated in other populations of Aplysia SNs (Hawkins et al. 1983; Walters and Byrne 1983; Abrams 1985; Ocorr et al. 1985; Yovell and Abrams 1992). However, a possible alternative explanation of the present results is that the effects of $\mathrm{SN}$ activity are mediated heterosynaptically. That is, if SNs synapse onto facilitatory interneurons, the effect of SN activation would simply be to increase the excitatory drive to the interneurons and thereby produce greater heterosynaptic facilitation onto the SNs. In this scenario, the enhanced facilitation produced by SN activity would presumably be widespread, rather than restricted to the activated neuron.

Two lines of evidence argue against this alternative explanation. First, the facilitation produced by $\mathrm{SN}$ activity has a different time course, with more rapid onset and decay, than heterosynaptic facilitation. Second, in the experiment examining receptive-field activation, shock within or outside the receptive of field of an individual $\mathrm{SN}$ should produce equivalent levels of activation of facilitatory interneurons. Therefore, heterosynaptic facilitation is not likely to account for the enhanced facilitation produced by shock within the receptive field of the SN. Thus, these findings support the hypothesis that SN activation induces an intrinsic, activity-dependent process of presynaptic facilitation that is specific to the activated SN. However, it has been reported recently that synapses of Aplysia sensory and MNs exhibit a form of homosynaptic facilitation that is similar to mammalian long-term potentiation in its requirement for postsynaptic depolarization (Lin and Glanzman 1994; Bao et al. 1997). In the present experiments, opportunity exists for postsynaptic activity to play a role, as tentacle shock stimuli elicit excitatory input to the postsynaptic MNs that were examined. Thus, in these experiments, the contribution of postsynaptic mechanisms to the induction of facilitation cannot be excluded.

\section{POSSIBLE CONTRIBUTION OF SN PLASTICITY TO NONCONTINGENT MODIFICATION OF HEAD-WAVING}

One objective of this investigation was to examine forms of SN plasticity that might be involved in noncontingent modification of head-waving behavior (Fitzgerald et al. 1997). The cellular experi-

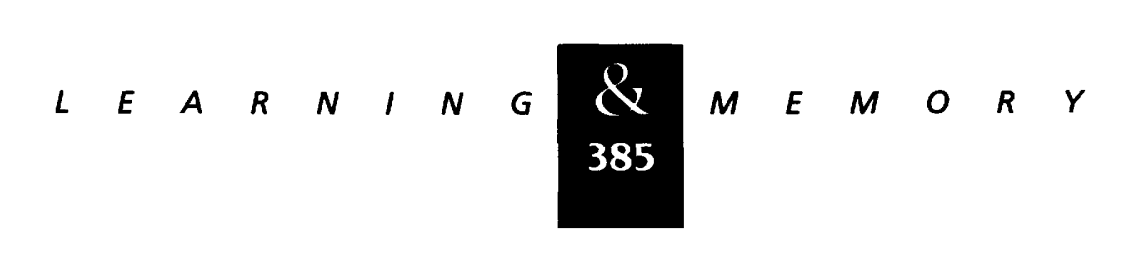


ments described here examined plasticity at the level of primary afferent neurons that receive input from the anterior tentacles. In reflexive behaviors, where SNs participate directly in driving the behavior, increases or decreases in SN synaptic output carry clear predictions for a resulting behavioral change. In the case of more complex behaviors such as head-waving, the functional role of SN plasticity is less obvious. Head-waving behavior is presumably generated by a central pattern generator, the circuitry of which is not yet known. Thus, the present data cannot provide a complete account of the mechanisms underlying learning expressed in head-waving. However, it is possible to identify several levels at which the kinds of neural plasticity that have been identified in this system could contribute to learning.

First, because the J/K SNs constitute a putative input pathway for aversive tentacle stimuli, plasticity in these neurons might serve to mediate US processing during training with multiple stimulus presentations. For example, heterosynaptic facilitation in these SNs induced by tentacle shock would be expected to amplify the effects of succeeding stimuli, thereby providing temporal summation within a series of stimuli. Along similar lines, activity-dependent synaptic facilitation would provide spatially restricted amplification of subsequent stimuli occurring at the same site (i.e., activating the same sensory afferents). Activity-dependent synaptic facilitation thus could provide a mechanism for the detection of spatial regularity of an aversive US. A behavioral prediction that arises from this concept is that an aversive US that is delivered consistently to the same location should be more effective (e.g., in inducing plasticity such as sensitization of withdrawal reflexes or modification of head-waving) than the same number of stimuli delivered in a spatially distributed manner.

In evaluating the present findings, it should be noted that the level of shock used to produce plasticity in the cellular experiments was higher than that used in the behavioral experiments. However, it is reasonable to expect that sensitivity to facilitating stimuli may be diminished in a reduced, previously anesthetized preparation. The present experiments examined synaptic and intrinsic $\mathrm{SN}$ plasticity using intracellularly evoked SN action potentials as test stimuli, an approach that enables controlled and quantitative measurements of synaptic strength and action potential dynamics. It is an interesting possibility for future investigation that other test stimuli, such as tactile activation of
SNs, might reveal additional forms of plasticity induced by tentacle shock (e.g., changes in SN threshold).

As described above, it is not evident how the activity or plasticity of cerebral ganglion SNs may relate to the generation or modulation of head-waving behavior. It is possible, however, to construct a hypothetical model of how the types of plasticity that have been demonstrated in cerebral ganglion SNs could contribute directly to changes in headwaving behavior. This model is based on the concept that activity-dependent facilitation at the synapses of SNs encodes the location of an aversive US presented during training. For facilitation to influence head-waving at these synapses, the SNs must be active during head-waving and must provide input to the circuitry producing the behavior. We therefore propose that afferent feedback, mediated at least in part by mechanoafferent SNs, participates in the generation of head-waving behavior; specifically, activity in SNs on one side of the body elicits a turning response away from that side. Our observations during the training phase of behavioral experiments suggest that Aplysia do in fact exhibit this kind of unconditioned response to anterior tentacle shock. Thus, in this model, learned modification of head-waving consists, at least in part, of a reflexive response elicited by afferent feedback, superimposed upon the head-waving pattern.

This model assumes that SNs are sensitive to sensory feedback during head-waving, such as the pressure produced by water currents during head movement. Although this level of tactile sensitivity has not been demonstrated empirically, two observations support the idea that either the $\mathrm{J} / \mathrm{K}$ cells or other SNs may mediate sensory feedback during head-waving. First, it is likely that the sensitivity of the $\mathrm{J} / \mathrm{K}$ SNs to tactile stimulation is considerably greater in the intact animal than in a reduced preparation. In the reduced preparation, manipulations such as stretching the skin or perfusing body structures with saline lower the tactile threshold of mechanoafferent SNs (Walters et al. 1983). Thus, in the behaving animal with an intact hydrostatic skeleton, the J/K SNs could likely exhibit greater sensitivity to tactile stimuli than has typically been observed in cellular experiments.

Second, it has been observed in the present experiments and elsewhere (Fredman and JahanParwar 1977) that the B cluster MNs receive excitatory synaptic input that is elicited by very light tactile stimulation of the anterior tentacles, includ-

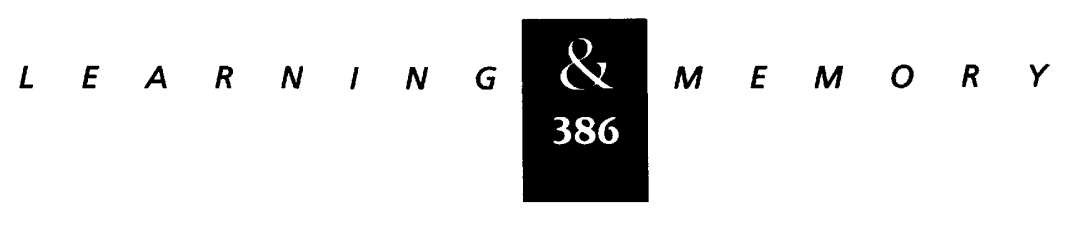


ing mere perturbation of the water near the tentacles without actual contact. The presence of this synaptic input implies that there are low-threshold primary afferents that innervate the anterior tentacles and produce synaptic input to the $B$ cells. Because none of the identified neurons in the cerebral ganglion appear to have response properties that match these characteristics, it is possible that the low-threshold afferents are located peripherally. Thus, the feedback function ascribed to the $\mathrm{J} / \mathrm{K} \mathrm{SNs}$ in this model might be mediated by a separate, parallel input pathway, possibly consisting of peripheral SNs.

Another possibility is that heterosynaptic, sidespecific plasticity, occurring at the level of a neural oscillator that generates the head-waving pattern, could account for learned changes in the behavioral output. For example, a half-center oscillator, consisting of bilateral, mutually inhibitory elements, may be involved in the production of headwaving. This type of oscillator has been found to underlie rhythmic behaviors in other systems, such as swimming in the mollusk Tritonia (Getting and Dekin 1985). Within this circuit, side-specific facilitation of the inhibitory synapse on one side would decrease activity in that pole of the oscillator and, thus, bias head-waving toward the opposite side. This model is structurally similar to that proposed by Raymond and colleagues (1992) to account for changes in head-waving produced by operant conditioning. Although these models are hypothetical in their details, they demonstrate ways in which known forms of plasticity could contribute to learning in the head-waving system and offer testable predictions for future exploration.

\section{Acknowledgments}

We thank Michael Davis, Edward Kairiss, Patricia Sharp, and Allan Wagner for helpful discussions of the work. This research was supported by National Institute of Mental Health grant $\mathrm{MH} 41083$ to T.J.C. and an National Science Foundation predoctoral fellowship to K.K.F.

The publication costs of this article were defrayed in part by payment of page charges. This article must therefore be hereby marked "advertisement" in accordance with 18 USC section 1734 solely to indicate this fact.

\section{References}

Abrams, T.W. 1985. Activity-dependent presynaptic facilitation: An associative mechanism in Aplysia. Cell. Mol. Neurobiol. 5: 123-145.

Bao, J.-X., E.R. Kandel, and R.D. Hawkins. 1997. Involvement of pre- and postsynaptic mechanisms in posttetanic potentiation at Aplysia synapses. Science 275: 969-973.

Byrne, J.H. and E.R. Kandel. 1996. Presynaptic facilitation revisited: State and time dependence. J. Neurosci. 16:425-435.

Byrne, J.H., D.A. Baxter, D.V. Buonomano, L.J. Cleary, A. Eskin, J.R. Goldsmith, E. McClendon, F.A. Nazif, F. Noel, and K.P. Scholz. 1991. Neural and molecular bases of nonassociative and associative learning in Aplysia. Ann. N.Y. Acad. Sci. 627: 124-49.

Castellucci, V.F. and S. Schacher. 1990. Synaptic plasticity and behavioral modifications in the marine mollusk Aplysia. Prog. Brain Res. 86: 105-15.

Clatworthy, A.L. and E.T. Walters. 1994. Comparative analysis of hyperexcitability and synaptic facilitation induced by nerve injury in two populations of mechanosensory neurones of Aplysia californica. J. Exp. Biol. 190: 217-238.

Fitzgerald, K., C.A. Takacs, and T.J. Carew. 1997. Nonassociative and associative modification of head-waving produced by aversive tentacular stimuli in Aplysia. Learn. \& Mem. (this issue).

Fredman, S.M. and B. Jahan-Parwar. 1977. Identifiable cerebral motoneurons mediating an anterior tentacular withdrawal reflex in Aplysia. J. Neurophysiol. 40: 608-615.

Getting, P.A. and M.S. Dekin. 1985. Tritonia swimming: A model system for integration within rhythmic motor systems. In Model neural networks and behavior (ed. A. I. Selverston), Plenum, New York, NY.

Hawkins, R.D. 1981. Interneurons involved in mediation and modulation of gill-withdrawal reflex in Aplysia. III. Identified facilitating neurons increase $\mathrm{Ca}^{2+}$ current in sensory neurons. J. Neurophysiol. 45: 327-339.

Hawkins, R.D. and S. Schacher. 1989. Identified facilitator interneurons $L 29$ and $L 28$ are excited by cutaneous stimuli used in dishabituation, sensitization, and classical conditioning of Aplysia. J. Neurosci. 9: 4236-4245.

Hawkins, R.D., T.W. Abrams, T.J. Carew, and E.R. Kandel. 1983. A cellular mechanism of classical conditioning in Aplysia: Activity-dependent amplification of presynaptic facilitation. Science 219: 400-404.

Klein, M. and E.R. Kandel. 1978. Presynaptic modulation of voltage-dependent $\mathrm{Ca}^{2+}$ current: Mechanism for behavioral sensitization in Aplysia californica. Proc. Natl. Acad. Sci. 75: 3512-3516.

Klein, M., J. Camardo, and E.R. Kandel. 1982. Serotonin modulates a specific potassium current in the sensory neurons that show presynaptic facilitation in Aplysia. Proc. Nat. Acad. Sci. 79: 5713-5717.

Lin, X.Y. and D.L. Glanzman. 1994. Long-term potentiation of Aplysia sensorimotor synapses in cell culture: Regulation by postsynaptic voltage. Proc. R. Soc. Lond. B 255: 113-118.

Ma, M. and J. Koester. 1996. The role of $\mathrm{K}^{+}$currents in frequency dependent spike broadening in Aplysia R20

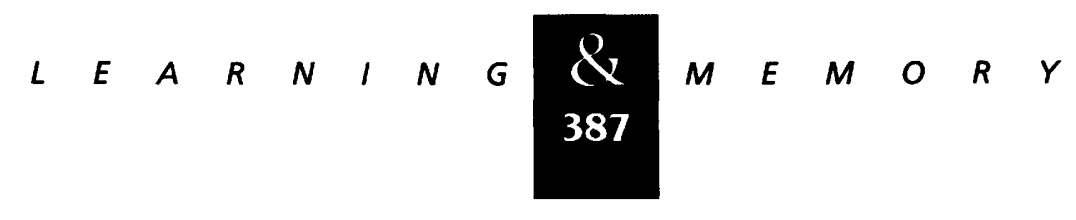


neurons: A dynamic clamp analysis. J. Neurosci. 16: 4089-4101.

Mackey, S.L., E.R. Kandel, and R.D. Hawkins. 1989. Identified serotonergic neurons LCB1 and RCB1 in the cerebral ganglia of Aplysia produce presynaptic facilitation of siphon sensory neurons. J. Neurosci. 9: 4227-4235.

Marcus, E.A., N.J. Emptage, R. Marois, and T.J. Carew. 1994. A comparison of the mechanistic relationships between development and learning in Aplysia. Prog. Brain Res. 100: $179-188$.

Ocorr, K.A., E.T. Walters, and J.H. Byrne. 1985. Associative conditioning analog selectively increase cAMP levels of tail sensory neurons on Aplysia. Proc. Natl. Acad. Sci. 82: 2548-2552.

Raymond, J.L. and J.H. Byrne. 1994. Distributed input to the tail-siphon withdrawal circuit in Aplysia from neurons in the ) cluster of the cerebral ganglion. J. Neurosci. 14: 2444-2454.

Raymond, J.L., D.A. Baxter, D.V. Buonomano, and J.H. Byrne. 1992. A learning rule based on empirically-derived activity-dependent neuromodulation supports operant conditioning in a small network. Neural Networks 5: 789-803.

Rosen, S.C., K.R. Weiss, and I. Kupfermann. 1979. Response properties and synaptic connections of mechanoafferent neurons in cerebral ganglion of Aplysia. J. Neurophysiol. 42: 954-974.

Rosen, S.C., K.R. Weiss, J.L. Cohen, and I. Kupfermann. 1982. Interganglionic cerebral-buccal mechanoafferents of Aplysia: Receptive fields and synaptic connections to different classes of neurons involved in feeding behavior. J. Neurophysiol. 48: 271-288.

Rosen, S.C., A.J. Susswein, E.C. Cropper, K.R. Weiss, and I. Kupfermann. 1989. Selective modulation of spike duration by serotonin and the neuropeptides, FMRFamide, $\mathrm{SCP}_{\mathrm{B}}$, buccalin and myomodulin in different classes of mechanoafferent neurons in the cerebral ganglion of Aplysia. J. Neurosci. 9: $390-402$.

Walters, E.T. 1987a. Site-specific sensitization of defensive reflexes in Aplysia: A simple model of long-term hyperalgesia. J. Neurosci. 7: 400-407.

$1987 \mathrm{~b}$. Multiple sensory neuronal correlates of site-specific sensitization in Aplysia. J. Neurosci. 7: 408-417.

Walters, E.T. and J.H. Byrne. 1983. Associative conditioning of single sensory neurons suggests a cellular mechanism for learning. Science 219: 405-408.

1984. Post-tetanic potentiation in Aplysia sensory neurons. Brain Res. 293: 377-380.

Walters, E.T., J.H. Byrne, T.J. Carew, and E.R. Kandel. 1983. Mechanoafferent neurons innervating tail of Aplysia. I.
Response properties and synaptic connections. J. Neurophysiol. 50: 1522-1542.

Yovell, Y. and T.W. Abrams. 1992. Temporal asymmetry in activation of Aplysia adenylyl cyclase by calcium and transmitter may explain temporal requirements of conditioning. Proc. Natl. Acad. Sci. 89: 6526-6530.

Received October 11, 1996; accepted in revised form February 3, 1997.

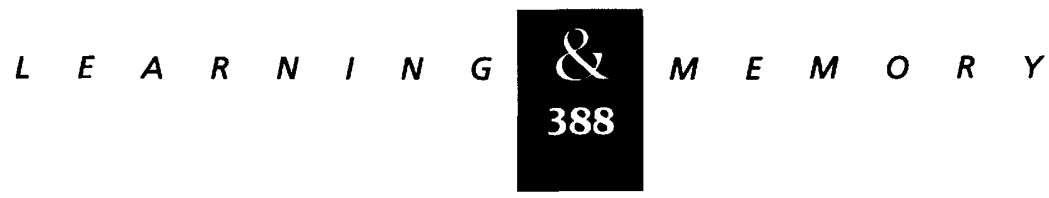




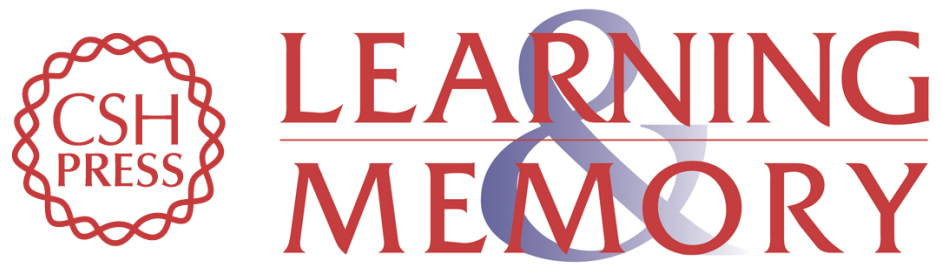

\section{Multiple forms of facilitation produced by aversive tentacular stimuli in cerebral ganglion sensory neurons of Aplysia.}

K K Fitzgerald and T J Carew

Learn. Mem. 1997, 3:

Access the most recent version at doi:10.1101//m.3.5.376

References This article cites 28 articles, 16 of which can be accessed free at: http://learnmem.cshlp.org/content/3/5/376.full.html\#ref-list-1

License

Email Alerting Receive free email alerts when new articles cite this article - sign up in the box at the Service top right corner of the article or click here. 